\title{
Depth-dependent target strengths of gadoids by the boundary- element method
}

\author{
David T. I. Francis \\ Department of Electronic, Electrical and Computer Engineering, University of Birmingham, Edgbaston, \\ Birmingham B15 2TT, United Kingdom
}

\author{
Kenneth G. Foote ${ }^{\mathrm{a})}$ \\ Woods Hole Oceanographic Institution, Woods Hole, Massachusetts 02543
}

(Received 5 October 2002; revised 24 July 2003; accepted 25 August 2003)

\begin{abstract}
The depth dependence of fish target strength has mostly eluded experimental investigation because of the need to distinguish it from depth-dependent behavioral effects, which may change the orientation distribution. The boundary-element method (BEM) offers an avenue of approach. Based on detailed morphometric data on 15 gadoid swimbladders, the BEM has been exercised to determine how the orientation dependence of target strength changes with pressure under the assumption that the fish swimbladder remains constant in shape and volume. The backscattering cross section has been computed at a nominal frequency of $38 \mathrm{kHz}$ as a function of orientation for each of three pressures: 1, 11, and 51 atm. Increased variability in target strength and more abundant and stronger resonances are both observed with increasing depth. The respective backscattering cross sections have been averaged with respect to each of four normal distributions of tilt angle, and the corresponding target strengths have been regressed on the logarithm of fish length. The tilt-angle-averaged backscattering cross sections at the highest pressure have also been averaged with respect to frequency over a $2-\mathrm{kHz}$ band for representative conditions of insonification. For all averaging methods, the mean target strength changes only slightly with depth. (c) 2003 Acoustical Society of America. [DOI: 10.1121/1.1619982]
\end{abstract}

PACS numbers: $43.30 . \mathrm{Gv}, 43.30 . \mathrm{Sf}[\mathrm{RAS}]$

Pages: $3136-3146$

\section{INTRODUCTION}

Numerous studies witness to the importance of fish target strength. ${ }^{1-5}$ This is, for example, a key quantity in the echo integration method of determining the numerical density of fish. ${ }^{6}$ It is also important in the echo counting method of density determination, as it appears in the samplingvolume term. ${ }^{7}$

In general, the target strength depends on fish size, orientation relative to the direction of incidence, acoustic frequency, biological state, depth, and depth history. A large number of studies have attempted to elucidate some of these dependences. References 4 and 8-12 are illustrative.

Determination of the depth dependence has been problematical for several reasons. In-situ observation based on free-swimming, unconfined fish is difficult for want of control over the fish, especially that of behavior through the orientation distribution, which may change with depth. When confined, the influence of captivity as a stressor must be suspected. The degree to which the fish controls the surface tension on the swimbladder wall may be significant, thus potentially affecting the target strength. ${ }^{13,14}$

Notwithstanding these comments, the depth dependence of target strength from swimbladdered fish lacking rete mirabile seems clear: Boyle's law, or the inverse relationship of ambient pressure and volume, is operative. The mass of gas in the swimbladder is constant, and the swimbladder volume

\footnotetext{
a) Author to whom correspondence should be addressed. Electronic mail: kfoote@whoi.edu
}

diminishes with depth, affecting the target strength. ${ }^{11}$ For swimbladdered fish possessing rete mirabile, the swimbladder volume can be regulated, presumably to maintain a state of fixed buoyancy independent of depth. Given the similar mass densities of fish flesh and sea water and similar compressibilities, such fish may be expected to maintain their swimbladders in a state of constant volume. If the swimbladder shape also remains constant, the target strength may be expected to remain more or less constant, with any difference in target strength being due to the increased mass density of gas with depth.

It is the present aim to investigate the effect of increased mass density of swimbladder gas with depth on the target strength of swimbladdered fish that maintain a constant gas volume. The particular fish are members of the gadoid family, specifically pollack (Pollachius pollachius) and saithe (Pollachius virens) for which the orientation dependence of target strength has already been measured ${ }^{15}$ and swimbladder morphometries, performed by Ona's method, ${ }^{16}$ are available. The approach is by theoretical modeling with the boundaryelement method, applied in a preceding study to the same specimens, but under assumption there of an empty swimbladder volume corresponding to an ideal pressure-release boundary. $^{17}$

\section{BOUNDARY-ELEMENT METHOD (BEM)}

In the acoustic boundary-element method, the Helmholtz equation $\left(\nabla^{2}+k^{2}\right) p=0$, where $k$ is the wavenumber, is recast as an integral equation in which the acoustic pressure $p$ 
at any point is expressed in terms of the pressure and normal displacement (or velocity) fields on the surface $S$ of the scatterer. ${ }^{18-20}$ To solve for the unknown fields, this integral equation is evaluated at each node associated with the set of discrete elements that collectively span $S$, thus producing a system of simultaneous equations for the pressures and displacements at those nodes. The solution to this system may then be used to determine the pressure at any other point using a numerical form of the original integral equation.

The exterior form of the standard integral equation is known to suffer from singularities at certain critical frequencies. ${ }^{21}$ Solutions to the integral equation may be nonunique. At critical frequencies, the aberrant solutions are physically inadmissible, ${ }^{22}$ which raises the problem of distinguishing these in numerical solutions.

Various methods exist to overcome the problem of critical frequencies, for example, those described in Refs. 23-28. The method available to the authors is based on Burton and Miller's approach, ${ }^{24}$ in the particular form developed in Ref. 29 and applied in Ref. 17. This incorporates a second integral equation, the normal derivative form, which is obtained by differentiating the standard form with respect to the normal direction at the surface. The two integral equations are combined by adding a multiple $\alpha$ of the normal derivative form to the standard form. The value of this coupling parameter was not specified by Burton and Miller; later authors have made various suggestions, e.g., Refs. 30-32, the consensus being that $\alpha$ should have a non-zero imaginary part and vary inversely as the wavenumber. Terai's recommendation ${ }^{31}$ that $\alpha=-\mathrm{i} / k$ has been adopted in the present work. The combined integral equation approach, in the particular formulation used here, is referred to as the partial Helmholtz gradient formulation ( $\mathrm{pHGF}$ ), while that of the standard integral equation is referred to simply as the standard formulation (SF).

Interestingly, inclusion of a fluid region interior to the scattering surface $S$, as in the current work, may be sufficient in itself to render the standard integral equation reliable at all frequencies, although this remains unproven. Because of the higher degrees of singularity of integrands in the pHGF, which are integrable, there is a nominal penalty to be paid: lesser precision relative to that achieved with the SF at noncritical frequencies.

To achieve the highest precision and accuracy, therefore, the pHGF is used to identify possible critical frequencies. In their apparent absence, the SF is used to describe the numerical results. The general pHGF is now introduced; the SF is derived from this by equating $\alpha$ to zero.

\section{A. Exterior equations}

The system of equations, in matrix form, is given by

$$
\mathbf{A p}=\mathbf{B u}-\mathbf{p}_{\text {inc }}-\alpha \frac{\partial \mathbf{p}_{\text {inc }}}{\partial n}
$$

where $\mathbf{p}$ is the pressure field and $\mathbf{u}$ is the normal component of the displacement field due to the incident pressure field $\mathbf{p}_{\text {inc }}$, with time variation $\exp (\mathrm{i} \omega t)$ understood, where $\omega$ is the angular frequency. The coefficients of the matrices $\mathbf{A}$ and $\mathbf{B}$ are assembled from local matrices pertaining to each element of the mesh. In performing the assembly, it is necessary to distinguish between the global label of a node, which is the label in the complete, global mesh, and the local node, which is the label of the node relative to an element on which it lies. With the calculation point of the Helmholtz integral taken at global node $i$, with position $\mathbf{r}_{i}$, integration over element $m$ provides the following coefficients in the standard formulation:

$$
\begin{aligned}
& a_{m n}^{(1)}\left(\mathbf{r}_{i}\right)=\int_{S_{m}} N_{n}(\mathbf{q}) \frac{\partial G\left(\mathbf{r}_{i}, \mathbf{q}\right)}{\partial n_{q}} \mathrm{~d} S_{q}, \\
& b_{m n}^{(1)}\left(\mathbf{r}_{i}\right)=\rho \omega^{2} \int_{S_{m}} N_{n}(\mathbf{q}) \cos \theta_{m n} G\left(\mathbf{r}_{i}, \mathbf{q}\right) \mathrm{d} S_{q},
\end{aligned}
$$

where $\rho$ is the fluid density, $\omega=c k, c$ is the speed of sound, $\mathbf{q}$ is the position vector of the integration point on the element surface $S_{m}, G$ is the Green's function, given by $G\left(\mathbf{r}_{i}, \mathbf{q}\right)=e^{-\mathrm{i} k\left|\mathbf{r}_{i}-\mathbf{q}\right|} / 4 \pi\left|\mathbf{r}_{i}-\mathbf{q}\right|, n$ is the local nodal label, and $N_{n}(\mathbf{q})(n=1,2, \ldots, 6$ for triangular elements, $n=1,2, \ldots, 8$ for quadrilateral elements) are the shape functions, which are of the standard second-order, or quadratic, form. ${ }^{33}$ The factor $\cos \theta_{m n}$ is included to allow for the deviation $\theta_{m n}$ of the normal to the element $m$ at local node $n$ from the mean normal at that node. This deviation is inherent in the quadratic formulation. The mean is weighted according to the differential area of each element at the node where they meet.

The normal derivative form of the Helmholtz integral equation, calculated at the centroids $\overline{\mathbf{r}}_{l}$ of the elements, similarly provides coefficients as follows:

$$
\begin{aligned}
& a_{m n}^{(2)}\left(\overline{\mathbf{r}}_{l}\right)=\int_{S_{m}} N_{n}(\mathbf{q}) \frac{\partial^{2} G\left(\overline{\mathbf{r}}_{l}, \mathbf{q}\right)}{\partial n_{r} \partial n_{q}} \mathrm{~d} S_{q}, \\
& b_{m n}^{(2)}\left(\overline{\mathbf{r}}_{l}\right)=\rho \omega^{2} \int_{S_{m}} N_{n}(\mathbf{q}) \cos \theta_{m n} \frac{\partial G\left(\overline{\mathbf{r}}_{l}, \mathbf{q}\right)}{\partial n_{r}} \mathrm{~d} S_{q},
\end{aligned}
$$

where the normal derivative is evaluated at the centroid. These are combined with the previous coefficients by adding a multiple of $a_{m n}^{(2)}\left(\overline{\mathbf{r}}_{l}\right)$ or $b_{m n}^{(2)}\left(\overline{\mathbf{r}}_{l}\right)$, respectively, for all elements $l$ on which global node $i$ lies, i.e.,

$$
\begin{aligned}
& a_{m n}\left(\mathbf{r}_{i}\right)=a_{m n}^{(1)}\left(\mathbf{r}_{i}\right)+\alpha_{i} \sum_{l: i \varepsilon S_{l}} a_{m n}^{(2)}\left(\overline{\mathbf{r}}_{l}\right), \\
& b_{m n}\left(\mathbf{r}_{i}\right)=b_{m n}^{(1)}\left(\mathbf{r}_{i}\right)+\alpha_{i} \sum_{l: i \varepsilon S_{l}} b_{m n}^{(2)}\left(\overline{\mathbf{r}}_{l}\right),
\end{aligned}
$$

where the combination factor $\alpha_{i}$ is taken to be $-\mathrm{i} / k M_{i}$, following Terai's recommendation, ${ }^{31}$ but allowing for the number $M_{i}$ of elements meeting at node $i{ }^{29-31}$

The coefficients $a_{m n}\left(\mathbf{r}_{i}\right)$ and $b_{m n}\left(\mathbf{r}_{i}\right)$ are assembled into the global matrices $\mathbf{A}$ and $\mathbf{B}$ by summing the coefficients that correspond to the same global node, thus

$$
\begin{aligned}
A_{i j} & =\sum_{m, n: C(m, n)=j} a_{m n}\left(\mathbf{r}_{i}\right)-\beta\left(\mathbf{r}_{i}\right) \delta_{i j}, \\
B_{i j} & =\sum_{m, n: C(m, n)=j} b_{m n}\left(\mathbf{r}_{i}\right),
\end{aligned}
$$

where $C(m, n)$ is the global node label of local node $n$ on element $m$. The quantity $4 \pi \beta(\mathbf{r})$ is the solid angle occupied 
by the fluid region surrounding the point $\mathbf{r} .{ }^{31}$ For $\mathbf{r}$ entirely within the fluid region, $\beta=1$; for $\mathbf{r}$ on the surface $S$ separating the fluid region from its neighbor, $\beta=0.5$ provided that $S$ is smooth at $\mathbf{r}$. For non-smooth surfaces such as those defined by the boundary-element meshes, methods of determining $\beta$ are described in Ref. 29.

The source terms in Eq. (1) are evaluated thus:

$$
\mathbf{p}_{\text {inc }}\left(\mathbf{r}_{i}\right)+\alpha_{i} \sum \frac{\partial p_{\text {inc }}\left(\overline{\mathbf{r}}_{l}\right)}{\partial n_{r}},
$$

where the summation is performed for all elements $l$ on which the global node $i$ lies.

\section{B. Interior equations}

The fundamental equation in the interior of the swimbladder resembles that of Eq. (1) but without the exciting pressure field $\mathbf{p}_{\text {inc }}$. Thus,

$$
\mathbf{A}_{1} \mathbf{p}-\mathbf{B}_{1} \mathbf{u}=0 .
$$

The matrices $\mathbf{A}_{1}$ and $\mathbf{B}_{1}$ resemble the respective matrices $\mathbf{A}$ and $\mathbf{B}$, but use the properties of the internal gas rather than those of the external fluid. In addition, the normal direction is oriented into the gas, hence it is reversed with respect to the normal direction in the exterior. The solid angle is similarly referred to the interior, hence

$$
\beta_{1}=1-\beta,
$$

at any point on $S$. With these conditions, a set of equations similar to those of Eqs. (2)-(5) can be developed.

\section{Simultaneous solution}

Pressure and normal displacement are continuous across the water-gas interface $S$, i.e., the nodal pressures and normal displacements in Eq. (1) are identical to those in Eq. (6). There are therefore as many unknown values of pressure and displacement as there are independent equations, namely $2 N$, where $N$ is the number of nodes on $S$. The simultaneous solution of Eqs. (1) and (6) is derived directly:

$$
\mathbf{u}=\left(\mathbf{B}-\mathbf{A} \mathbf{A}_{1}^{-1} \mathbf{B}_{1}\right)^{-1}\left(\mathbf{p}_{\text {inc }}+\alpha \frac{\partial \mathbf{p}_{\text {inc }}}{\partial n}\right),
$$

and

$$
\mathbf{p}=\mathbf{A}_{1}^{-1} \mathbf{B}_{1} \mathbf{u} .
$$

\section{Scattered field}

The scattered pressure at an exterior point $\mathbf{r}$ is obtained from the standard integral equation by calculating coefficients similar to $a_{m n}^{(1)}\left(\mathbf{r}_{i}\right)$ and $b_{m n}^{(1)}\left(\mathbf{r}_{i}\right)$, but with $\mathbf{r}_{i}$ replaced by the position vector $\mathbf{r}$ :

$$
\begin{aligned}
& a_{j}^{(3)}(\mathbf{r})=\sum_{m, n: C(m, n)=j} \int_{S_{m}} N_{n}(\mathbf{q}) \frac{\partial G(\mathbf{r}, \mathbf{q})}{\partial n_{q}} \mathrm{~d} S_{q}, \\
& b_{j}^{(3)}(\mathbf{r})=\rho \omega^{2} \sum_{m, n: C(m, n)=j} \int_{S_{m}} N_{n}(\mathbf{q}) \cos \theta_{m n} G(\mathbf{r}, \mathbf{q}) \mathrm{d} S_{q},
\end{aligned}
$$

and then

$$
p(\mathbf{r})=\mathbf{a}^{(3)}(\mathbf{r}) \cdot \mathbf{p}-\mathbf{b}^{(3)}(\mathbf{r}) \cdot \mathbf{u},
$$

where the dot-product operations are indicated. The backscattering amplitude at finite range $r$ is

$$
f(r)=\frac{r|p(\mathbf{r})|}{\left|p_{\text {ind }}\right|} .
$$

The farfield backscattering amplitude $f$ is the limit of $f(r)$ as $r$ approaches infinity. Expressions for the backscattering cross section $\sigma$ and target strength TS are derived by substituting $f(r)$, or $f$, in the following equations:

$$
\sigma=4 \pi|f|^{2}
$$

and

$$
\mathrm{TS}=10 \log \left[\frac{\sigma}{4 \pi r_{0}^{2}}\right],
$$

where $r_{0}$ is a reference distance, assumed here to be $1 \mathrm{~m}$.

\section{E. Numerical evaluation techniques}

The elements used here are quadrilaterals and triangles of the quadratic isoparametric type, in which both the geometric and acoustic quantities are interpolated from the nodal values using quadratic shape functions, the nodes being situated at the vertices and mid-sides. ${ }^{33}$ Experience suggests that good representation of the acoustic variables is obtained if the lengths of the sides of the elements are less than onethird of a wavelength. ${ }^{17}$ The accuracy of geometrical representation depends on the degree of undulation of the surface, but it should be noted that the quadratic interpolation allows the sides and faces of the elements to be curved. Further details of the formulation and equations can be found in Ref. 29.

In evaluating the coefficients in Eqs. (1) and (6), Gauss quadrature is used.

\section{INDEPENDENT VALIDATION OF BEM}

The BEM has been validated previously for application to surface-adapted gadoids, but assuming that the swimbladder acts as a void. ${ }^{17}$ The BEM was also tested against the example of scattering by a spherical void for which a series solution is available.

For application to problems of scattering by a swimbladdered fish at depth, the method is tested against the analytical (series) solutions ${ }^{34,35}$ for two test cases. The object in both cases is a constant-volume gas-filled sphere of diameter 50 $\mathrm{mm}$. In the first case, bistatic scattering for an incident wave of frequency $50 \mathrm{kHz}$ is described over the angular range $180^{\circ}$ as measured from the forward direction at each of two pressures, 1 and $51 \mathrm{~atm}$, corresponding to the nominal depths of 0 and $500 \mathrm{~m}$. The values of density $\rho$ and sound speed $c$ were taken to be $1025 \mathrm{~kg} / \mathrm{m}^{3}$ and $1470 \mathrm{~m} / \mathrm{s}$, respectively, in water; $1.247 \mathrm{~kg} / \mathrm{m}^{3}$ and $337.4 \mathrm{~m} / \mathrm{s}$, respectively, in air at 1 atm; and $63.597 \mathrm{~kg} / \mathrm{m}^{3}$ and $337.4 \mathrm{~m} / \mathrm{s}$, respectively, in air at $51 \mathrm{~atm}$. The mass density of swimbladder gas is assumed to change in proportion to pressure to maintain a constant volume. For the BEM, the mesh representing the sphere was formed from elements delineated by lines of latitude and 


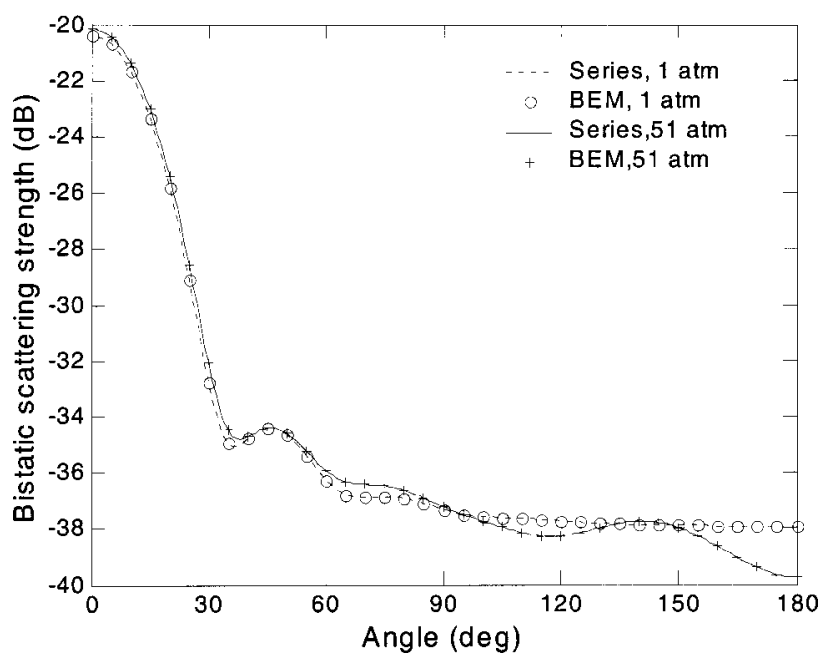

FIG. 1. Bistatic scattering strength in decibels as a function of scattering angle for a plane wave of frequency $50 \mathrm{kHz}$ incident on a spherical air bubble of diameter $50 \mathrm{~mm}$, at pressures of 1 and $51 \mathrm{~atm}$. The analytical (series) solution is shown by the continuous and broken lines, while the BEM predictions are shown as discrete points. The scattering angle is relative to the direction of incidence, so that $0^{\circ}$ represents the forward direction.

longitude at $2.25^{\circ}$ intervals. By invoking rotational symmetry, the problem was reduced to one of solving for the surface pressures and displacements on only one segment, consisting of 80 elements between adjacent meridians. The results shown in Fig. 1 indicate an excellent agreement between the
BEM and the analytical solution. Results with the pHGF and SF are indistinguishable.

In the second test case, the target strength of a similar gas-filled sphere is computed over the frequency range $1 \mathrm{~Hz}$ to $50 \mathrm{kHz}$, again at pressures of 1 and $51 \mathrm{~atm}$, with densities and sound speeds as given above. The target strength was initially calculated using the analytical solution at $1-\mathrm{Hz}$ intervals. In the neighborhood of each of the numerous peaks and troughs identified from these initial results, further calculations were then performed at increasing resolution, down to $0.001 \mathrm{~Hz}$ as required, in order to identify the peak or trough TS values to within $0.1 \mathrm{~dB}$. For the BEM, the longer computing times made it impractical to compute solutions to the same resolution of $1 \mathrm{~Hz}$ over the whole frequency range. Instead, the target strength was calculated initially at $100-\mathrm{Hz}$ intervals, and then at finer resolution around the peaks and troughs as identified in the analytical solution, again with the aim of determining the predicted peak or trough TS value to within $0.1 \mathrm{~dB}$.

The same mesh as described in the first test case was used in the frequency range $25-50 \mathrm{kHz}$. For lower frequencies, where the condition that the nodal separation should be less than $\frac{1}{6}$ of the wavelength allows for larger elements, similar meshes were used but at intervals in latitude and longitude of $4.5^{\circ}$ for the range $12.5-25 \mathrm{kHz}$ and $9^{\circ}$ for the range $0-12.5 \mathrm{kHz}$.

The results are plotted in Fig. 2(a) for a pressure of 1

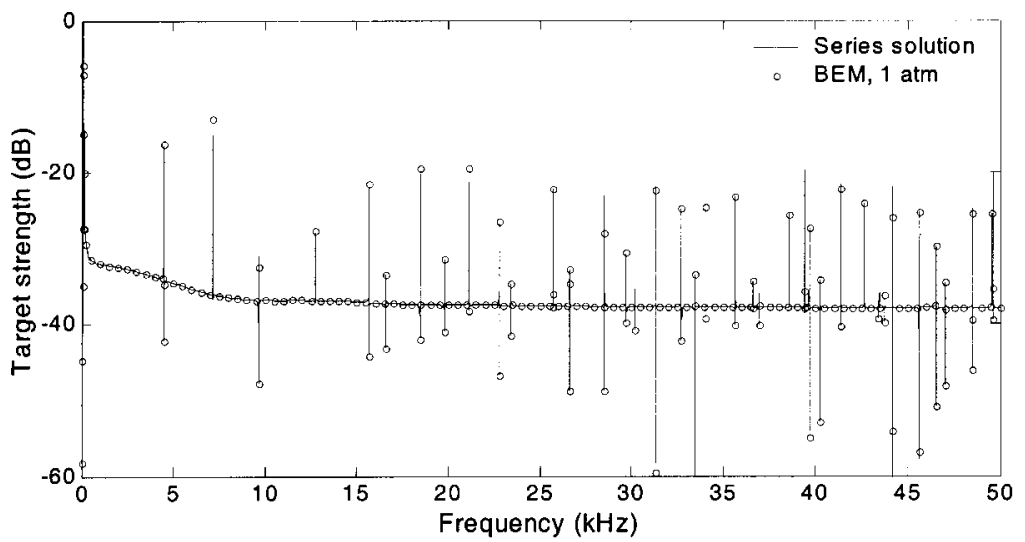

(a)

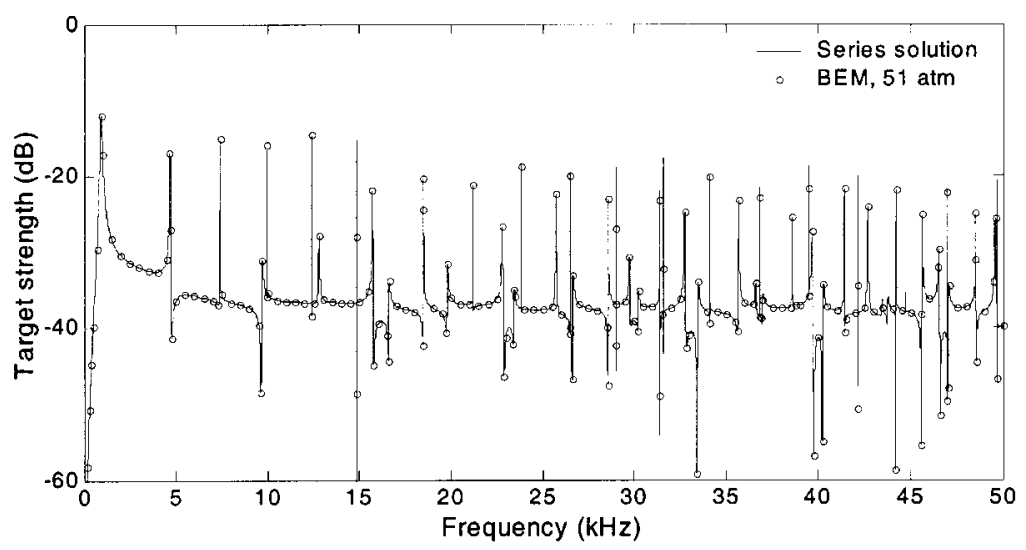

(b)
FIG. 2. Target strength of a spherical air bubble of diameter $50 \mathrm{~mm}$ in water at (a) $1 \mathrm{~atm}$ and (b) $51 \mathrm{~atm}$. The analytical solution is shown by a continuous line, the BEM predictions as discrete points. In (a), the first peak, at $0.13 \mathrm{kHz}$ with a TS of $5.1 \mathrm{~dB}$, is truncated. 
TABLE I. Properties of the 15 swimbladders and the meshes used to represent them.

\begin{tabular}{|c|c|c|c|c|c|c|c|c|c|c|}
\hline \multirow[b]{2}{*}{ Fish no. } & \multirow[b]{2}{*}{ Species } & \multirow{2}{*}{$\begin{array}{c}\text { Fish } \\
\text { length } \\
(\mathrm{cm})\end{array}$} & \multirow{2}{*}{$\begin{array}{c}\text { Fish } \\
\text { mass } \\
(\mathrm{g})\end{array}$} & \multicolumn{2}{|c|}{ Meshes } & \multicolumn{2}{|c|}{ Swimbladder } & \multicolumn{3}{|c|}{ Nodal separation } \\
\hline & & & & $n_{\text {elem }}$ & $n_{\text {nodes }}$ & $\begin{array}{c}\text { Surface } \\
\text { area }\left(\mathrm{cm}^{2}\right)\end{array}$ & $\begin{array}{c}\text { Volume } \\
\left(\mathrm{cm}^{3}\right)\end{array}$ & $\begin{array}{l}95 \% \text { limit } \\
(\mathrm{cm})\end{array}$ & $\begin{array}{l}99 \% \text { limit } \\
(\mathrm{cm})\end{array}$ & $\begin{array}{l}\operatorname{Max} \\
(\mathrm{cm})\end{array}$ \\
\hline 201 & Pollack & 31.5 & 195 & 1168 & 3364 & 33.01 & 6.91 & 0.111 & 0.120 & 0.221 \\
\hline 202 & Pollack & 44.0 & 533 & 1389 & 4041 & 58.83 & 16.33 & 0.126 & 0.137 & 0.177 \\
\hline 204 & Pollack & 35.5 & 321 & 1078 & 3116 & 42.39 & 10.03 & 0.131 & 0.141 & 0.172 \\
\hline 205 & Pollack & 39.0 & 380 & 1107 & 3181 & 45.75 & 11.34 & 0.132 & 0.143 & 0.193 \\
\hline 206 & Pollack & 35.0 & 287 & 1159 & 3347 & 31.37 & 7.75 & 0.104 & 0.117 & 0.146 \\
\hline 207 & Pollack & 44.5 & 635 & 1487 & 4363 & 65.24 & 19.15 & 0.124 & 0.134 & 0.161 \\
\hline 209 & Saithe & 38.5 & 385 & 1501 & 4387 & 43.29 & 10.08 & 0.100 & 0.106 & 0.139 \\
\hline 213 & Pollack & 34.5 & 259 & 1039 & 2935 & 34.11 & 7.83 & 0.123 & 0.133 & 0.161 \\
\hline 214 & Pollack & 39.0 & 406 & 1164 & 3362 & 44.14 & 10.15 & 0.125 & 0.134 & 0.153 \\
\hline 215 & Pollack & 37.0 & 332 & 1076 & 3092 & 38.89 & 8.75 & 0.124 & 0.134 & 0.174 \\
\hline 216 & Pollack & 36.5 & 343 & 1062 & 3060 & 43.33 & 10.85 & 0.131 & 0.140 & 0.164 \\
\hline 217 & Pollack & 34.5 & 253 & 1662 & 4840 & 32.15 & 6.57 & 0.081 & 0.088 & 0.134 \\
\hline 218 & Pollack & 32.5 & 257 & 1327 & 3879 & 29.75 & 6.27 & 0.092 & 0.100 & 0.139 \\
\hline 219 & Pollack & 35.5 & 292 & 1039 & 3005 & 35.74 & 8.15 & 0.120 & 0.127 & 0.153 \\
\hline 220 & Saithe & 38.0 & 406 & 1321 & 3857 & 44.32 & 10.46 & 0.106 & 0.113 & 0.132 \\
\hline
\end{tabular}

atm, and Fig. 2(b) for a pressure of 51 atm. For clarity, the BEM predictions, shown as discrete points, are plotted at intervals of $500 \mathrm{~Hz}$, with the addition of the peak and trough values. The SF results are shown for giving slightly better agreement with the exact series solution. This agreement is excellent away from the peaks and in the values of the frequencies at which the peaks and troughs occur; the main discrepancies occur in the predictions of the peak and trough values at high- $Q$ resonances. At these frequencies the system of equations solved in Eq. (8) may be less well conditioned, and numerical errors in the coefficients and in the solution of the system of equations become more significant.

While the original purpose of this test case was to investigate the performance of the BEM, the results in Fig. 2 provide insight into the effect of pressure on the target strength response when the interior fluid is included in the analysis. No resonances appear when the bubble is treated as a void, which is illustrated in Ref. 17, Fig. 2. At 1 atm, resonances appear, but with very narrow bandwidths, typically less than $1 \mathrm{~Hz}$ for the case considered. These bandwidths increase with increasing pressure. At $51 \mathrm{~atm}$, there is a notable effect, with deviations from the smooth line of the solution for a void in the frequency bands between neighboring resonances.

\section{SWIMBLADDER MORPHOMETRY}

The swimbladder morphometric data were derived from a study performed in 1980 on surface-adapted gadoids ${ }^{15}$ by Ona's method of cryomicrotoming. ${ }^{16}$ The data were reduced to a set of curvilinear quadrilaterals and triangles spanning each swimbladder surface, as described in Ref. 17. The statistics of the nodes at which the fundamental equations (8) and (9) were solved are described in Table I, which also summarizes information on the specimens themselves: species, length, mass, and so forth. An example of one of the meshes, that for specimen $217,{ }^{36}$ is shown in Fig. 3.

As earlier noted, ${ }^{17}$ the BEM is considered valid for nodal spacings less than one-sixth of the acoustic wavelength. It is important to note that, in the case of gas-filled swimbladders, the relevant wavelength is that of the acoustic field inside the swimbladder. Relative to the wavelength $\lambda$ in the exterior immersion medium with sound speed $c$, the interior wavelength $\lambda_{1}$ is

$$
\lambda_{1}=\frac{\lambda c_{1}}{c},
$$

where $c_{1}$ is the speed of sound in the gas. Thus, whereas the nodal spacings were adequate for computations at $120 \mathrm{kHz}$ in the preceding work on scattering by voids, the nodal spacings are only adequate here for computations up to about 40 $\mathrm{kHz}$.

\section{COMPUTATIONAL PARAMETERS}

The mass density of the gas in the swimbladder at atmospheric pressure is assumed to be that of air at standard condition, namely $1.247 \mathrm{~kg} / \mathrm{m}^{3}$. At ambient pressure $P$ atm, the mass density is assumed to be $1.247 P \mathrm{Pg} / \mathrm{m}^{3}$. The speed of sound in the gas is assumed to be $337.4 \mathrm{~m} / \mathrm{s}$, independent of depth.

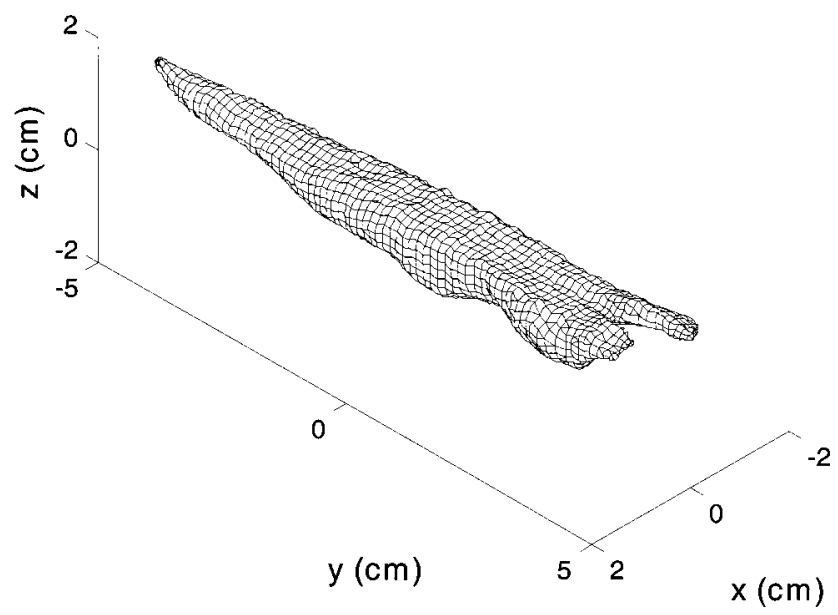

FIG. 3. Boundary-element mesh of the swimbladder to specimen 217 of Ref. 36. The meshed swimbladder length is $108 \mathrm{~mm}$ and the mesh has 1662 elements and 4840 nodes. 
The mass density of the surrounding fish flesh is assumed to be identical to that of the seawater immersion medium, namely $1025 \mathrm{~kg} / \mathrm{m}^{3}$. For the assumed temperature of $5{ }^{\circ} \mathrm{C}$ and salinity of $35 \mathrm{ppt}$, the nominal sound speed in the immersion medium is $1470 \mathrm{~m} / \mathrm{s}$.

The numbers of elements and nodes for each swimbladder are given in Table I. Statistical measures of the distance between nearest-neighbor nodes are given in the same table. As mentioned, this limits the upper frequency of computation to about $40 \mathrm{kHz}$, on the basis that the length of element sides, nominally double the nodal separation, should be less than one-third of a wavelength in air.

The backscattering cross section given by Eq. (13) is averaged with respect to the orientation distribution, then expressed in the logarithmic domain according to Eq. (14). Each of four normal distributions of tilt angle are considered: $\mathrm{N}(0,0), \mathrm{N}(0,5), \mathrm{N}(0,10)$, and $\mathrm{N}(-4.4,16)^{\circ}$. The last distribution is derived from in-situ photographic observations on cod (Gadus morhua). ${ }^{37}$

Because of the effect of perspective, ${ }^{38}$ the effective standard deviations for the four cases are 2.5, 5.5, 10.2, and $16^{\circ}$, respectively. ${ }^{36}$ In terms of the normal distribution $g$ of tilt angle $\theta$, with mean $\bar{\theta}$ and effective standard deviation $s_{\theta}$, the average backscattering cross section at frequency $\nu$ is

$$
\bar{\sigma}(\nu)=\int g(\theta) \sigma(\nu, \theta) d \theta / \int g(\theta) d \theta,
$$

where the integration is performed over the range $[\bar{\theta}$ $\left.-3 s_{\theta}, \bar{\theta}+3 s_{\theta}\right]$.

Because of the presence of gas in the swimbladder cavity, resonances occur, even at high frequencies beyond the low-frequency breathing-mode resonance. ${ }^{39}$ These could be troublesome, particularly at greater depths since the greater bandwidths of the resonances, observed in the results for the spherical bubble, make it more likely that a particular frequency will fall within such a resonance. However, there are no infinite-duration, single-frequency signals in practice; sonar measurements are performed with finite-duration signals, hence with bandwidth. Realizable receivers also have an associated bandwidth of processing. These effects have been dealt with in Ref. 40 through the following operational average of the backscattering cross section as a function of tilt angle $\theta$ for a downward-pointing transducer, here expressed in terms of the tilt-angle-averaged cross section:

$$
\overline{\bar{\sigma}}=\int|S H|^{2} \bar{\sigma}(\nu) d \nu / \int|S H|^{2} d \nu
$$

where $S$ is the transmit signal spectrum and $H$ is the receiver frequency response function, all functions of frequency $\nu$. In the computations reported here, $S$ is the Fourier spectrum corresponding to the signal $s(t)=\cos \left(2 \pi \nu_{0} t\right) \operatorname{rect}(t / \tau)$, where $\nu_{0}$ is the center frequency of the transmit signal, $\tau$ is the signal duration, assumed to be $0.64 \mathrm{~ms}$, and $\operatorname{rect}(x)$ is 0 for $|x|>0.5$ and 1 for $|x| \leqslant 0.5$. Thus, $S(\nu)=(\sin y) / y$, where $y$ $=\left(\nu-\nu_{0}\right) \tau / 2$. The function $H$ is described in Fig. 4 for $\nu_{0}$ $=38 \mathrm{kHz}$.

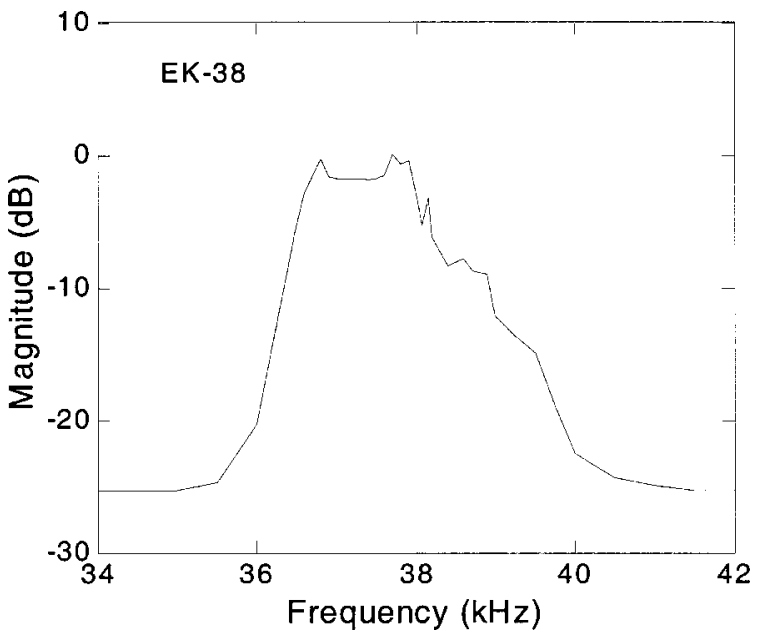

FIG. 4. Magnitude of the frequency response function of the receiver of the EK-38 echo sounder, from Fig. 4 of Ref. 40.

\section{RESULTS AND DISCUSSION}

\section{A. Comparison of computations with standard and partial Helmholtz gradient formulations}

Results with the two approaches are illustrated in Fig. 5 for specimens 217 and 219. The resolution of the pHGF results is $50 \mathrm{~Hz}$; that of the SF results is $25 \mathrm{~Hz}$. Evidently, the agreement is very strong, but with a few differences where the pHGF results appear to be slightly more dispersed, suggesting a somewhat greater variability. As mentioned in Sec. II, this is believed to be due to the higher degrees of singularity of integrands in the pHGF compared with those in the SF, witness Eq. (3a) compared with Eq. (2b), with corresponding loss of numerical precision. By reason of assumed greater precision and accuracy, the SF is used to derive the computational results presented below.

Agreement of the corresponding results for the two approaches is poorer for some other specimens, especially when their nodal spacing approaches the $\lambda / 6$-limit of applicability, where $\lambda$ is the wavelength of sound in the gaseous interior of the swimbladder. At $38.1 \mathrm{kHz}$, with speed of sound in the gas of $337.4 \mathrm{~m} / \mathrm{s}, \lambda / 6=0.144 \mathrm{~cm}$. Referring to Table I, 95\% of the nodal spacings for specimen no. 219 are less than $0.120 \mathrm{~cm}$. This is within $10 \%$ of the corresponding value for nodal spacing with the coarsest mesh, which applies to specimen 205.

\section{B. Depth dependence of target strength for a single specimen}

The tilt-angle dependence of target strength has been computed for each specimen at each of three pressures: 1,11 , and $51 \mathrm{~atm}$. The dependences for the swimbladder modeled as a void ${ }^{17}$ and at pressures of 1 and 11 atm are in very close agreement.

The computed tilt-angle dependence of target strength is shown for a single specimen, a $34.5-\mathrm{cm}$-long pollack, in Fig. 6. Also shown are the dependences for the case of a void of the same shape ${ }^{17}$ and for actual measurements of the whole fish with intact swimbladder. ${ }^{36}$ 

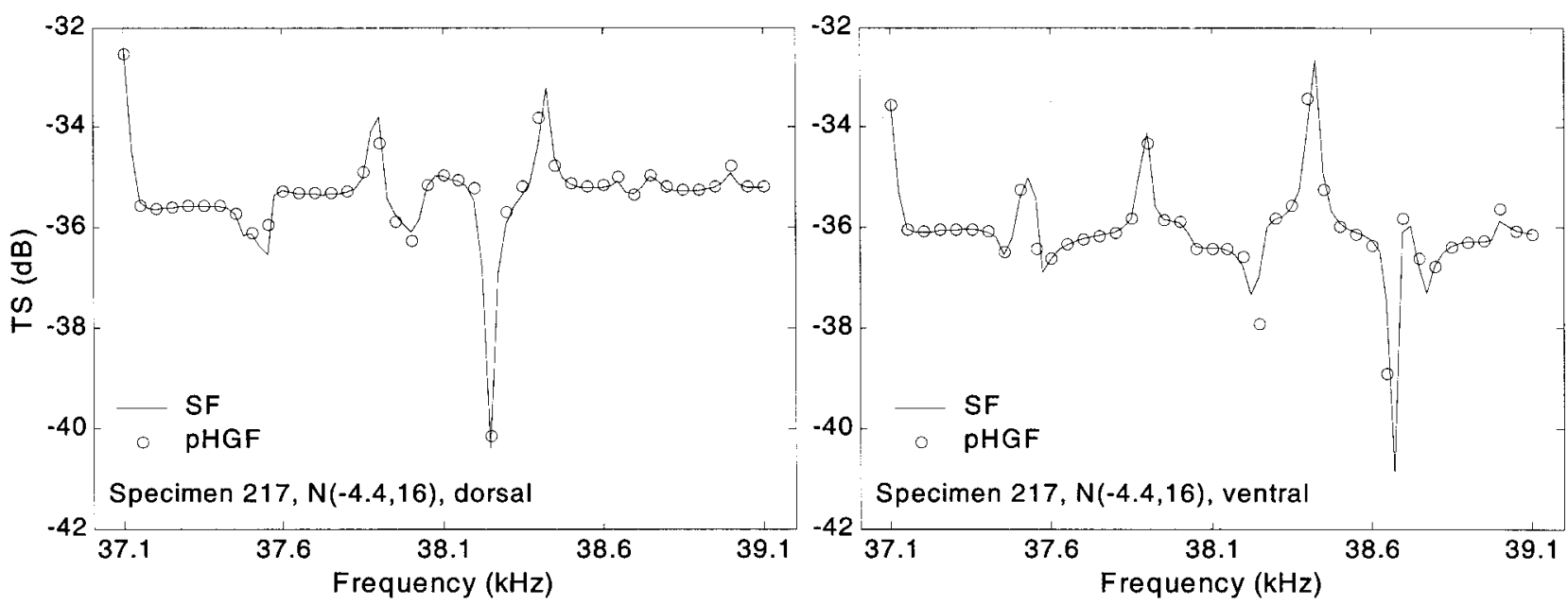

(a)
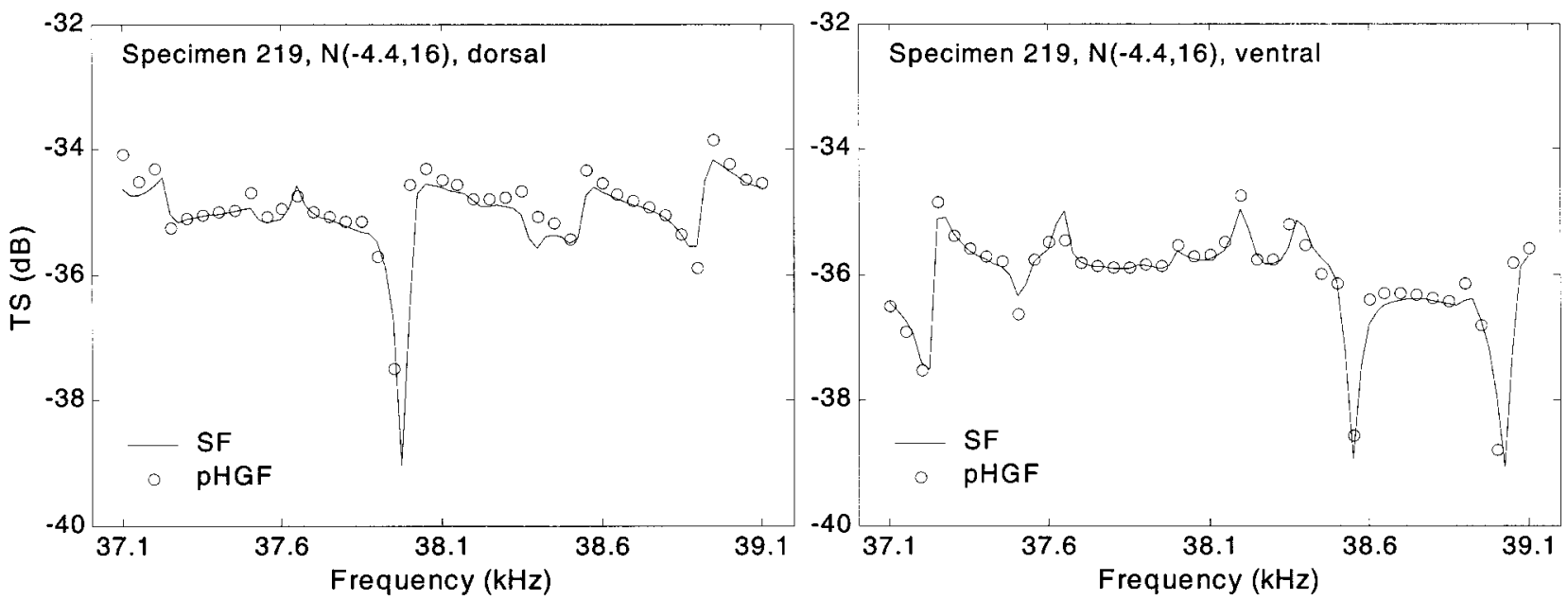

(b)

FIG. 5. Predicted target strength versus frequency for (a) specimen 217 and (b) specimen 219 in the range $37.1-39.1 \mathrm{kHz}$, at a pressure of $51 \mathrm{~atm}$. Results obtained using the standard formulation (SF) of the BEM, at intervals of $25 \mathrm{~Hz}$, are shown by the continuous lines; results from the partial Helmholtz gradient formulation (pHGF) are shown as discrete points, at intervals of $50 \mathrm{~Hz}$.

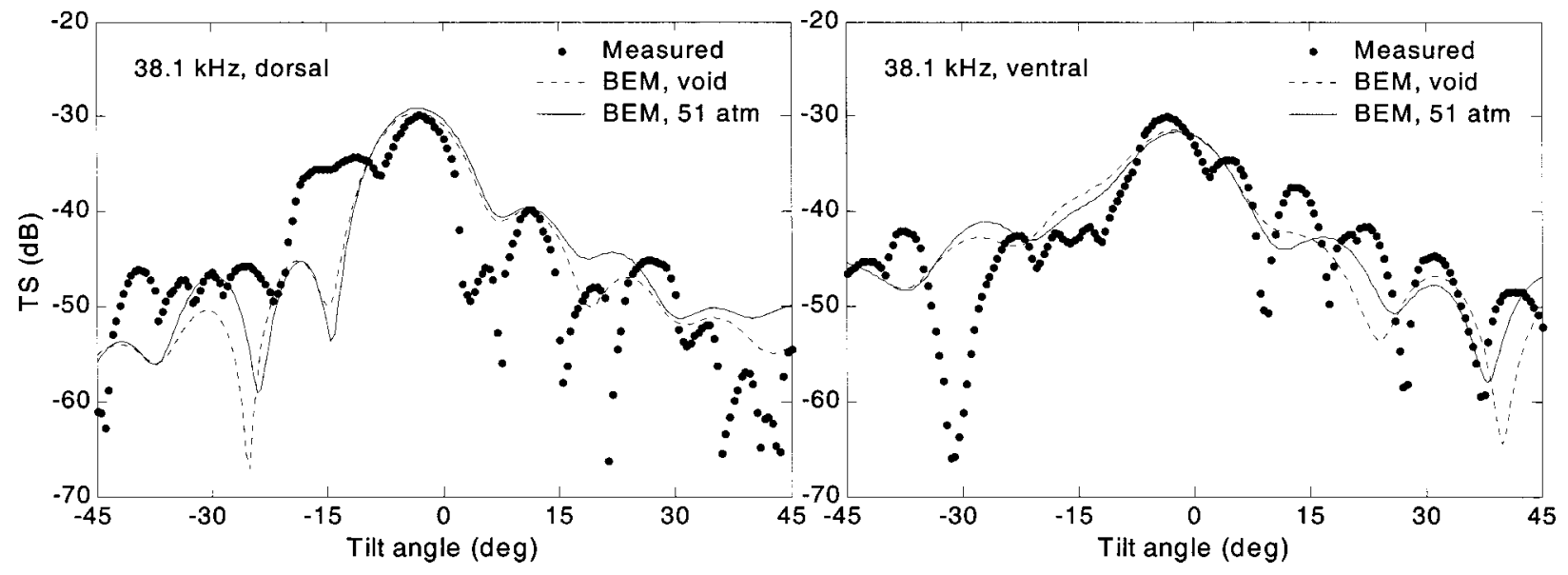

FIG. 6. BEM computations of target strength as a function of tilt angle for swimbladder specimen 217 , treated as a void and as gas-filled at a depth of 500 $\mathrm{m}$, compared against direct measurements at $2.5-\mathrm{m}$ depth. The functions are shown for both dorsal and ventral aspects at a frequency of $38.1 \mathrm{kHz}$. 
TABLE II. Regression analyses of target strength on fish length, based on BEM computations of TS for 15 specimens in dorsal aspect. Averaging is performed with respect to each of four normal distributions of tilt angle at each of four pressures: $0,1,11$, and 51 atm. The tilt-angle-averaged results at 51 atm are additionally averaged with respect to frequency, using Eq. (17), and presented in the final row for each tilt-angle distribution. Results are expressed through the regression coefficient $b$ in Eq. (18) and standard error SE of the regression. The correlation coefficient $\rho$ of underlying target strength values in each set is also shown.

\begin{tabular}{|c|c|c|c|c|c|c|}
\hline \multirow{2}{*}{$\begin{array}{c}\text { Frequency } \\
\mathrm{kHz}\end{array}$} & \multicolumn{2}{|c|}{ Tilt angle (degrees) } & \multirow{2}{*}{$\begin{array}{c}\text { Pressure } \\
\text { (atm) }\end{array}$} & \multicolumn{3}{|c|}{ BEM computed TS functions } \\
\hline & Mean & s.d. & & $\rho$ & $b$ & SE \\
\hline 38.1 & 0.0 & 0.0 & 0 & 0.933 & -61.36 & 1.00 \\
\hline 38.1 & 0.0 & 0.0 & 1 & 0.933 & -61.36 & 1.00 \\
\hline 38.1 & 0.0 & 0.0 & 11 & 0.930 & -61.38 & 1.00 \\
\hline 38.1 & 0.0 & 0.0 & 51 & 0.747 & -61.30 & 1.37 \\
\hline $37.1-39.1$ & 0.0 & 0.0 & 51 & 0.922 & -61.46 & 1.00 \\
\hline 38.1 & 0.0 & 5.0 & 0 & 0.945 & -62.44 & 0.66 \\
\hline 38.1 & 0.0 & 5.0 & 1 & 0.945 & -62.44 & 0.65 \\
\hline 38.1 & 0.0 & 5.0 & 11 & 0.938 & -62.45 & 0.66 \\
\hline 38.1 & 0.0 & 5.0 & 51 & 0.709 & -62.40 & 1.09 \\
\hline $37.1-39.1$ & 0.0 & 5.0 & 51 & 0.930 & -62.55 & 0.67 \\
\hline 38.1 & 0.0 & 10.0 & 0 & 0.946 & -64.18 & 0.47 \\
\hline 38.1 & 0.0 & 10.0 & 1 & 0.947 & -64.18 & 0.46 \\
\hline 38.1 & 0.0 & 10.0 & 11 & 0.938 & -64.19 & 0.47 \\
\hline 38.1 & 0.0 & 10.0 & 51 & 0.664 & -64.14 & 0.98 \\
\hline $37.1-39.1$ & 0.0 & 10.0 & 51 & 0.930 & -64.28 & 0.49 \\
\hline 38.1 & -4.4 & 16.0 & 0 & 0.941 & -65.76 & 0.40 \\
\hline 38.1 & -4.4 & 16.0 & 1 & 0.942 & -65.76 & 0.39 \\
\hline 38.1 & -4.4 & 16.0 & 11 & 0.931 & -65.76 & 0.41 \\
\hline 38.1 & -4.4 & 16.0 & 51 & 0.610 & -65.68 & 0.96 \\
\hline $37.1-39.1$ & -4.4 & 16.0 & 51 & 0.924 & -65.81 & 0.43 \\
\hline
\end{tabular}

The effect of pressure on the orientation dependence of target strength of the other 14 specimens is similar, if differing in the precise details of deviations from the respective void case and measurements.

\section{Target strength-length regressions}

The large number of computations that have been performed for the 15 swimbladders have been combined by averaging. In particular, the backscattering cross section computed with the BEM, and illustrated in Fig. 6 for a single specimen, have been averaged with respect to the tilt-angle distributions according to Eq. (16). The target strength corresponding to $\bar{\sigma}$ has then been computed according to Eq. (14), and the regression equation

$$
\mathrm{TS}=20 \log l+b
$$

computed by the method of least squares, where $l$ is the total fish length in centimeters. Results are shown in Table II for dorsal aspect and in Table III for ventral aspect. Scatter diagrams of target strength and fish length have been prepared for the tilt-angle distribution $\mathrm{N}(-4.4,16)^{\circ}$ for a void and at a pressure of $51 \mathrm{~atm}$. They are presented in Fig. 7.

In general, the modeled target strengths display increasing variability with increasing depth. The physical explanation for this is presently unclear, but based on the second validation example, in Fig. 2, with evidence of resonances at frequencies above the ordinary very-low-frequency breathing-mode resonance, it is speculated that stronger resonances are excited more easily at greater pressures. Thus, TS values are likely to be elevated or depressed depending on the proximity of a resonance or anti-resonance, respectively, for each specimen. Physically, the potential of the swimbladder under pressure to store acoustic energy and act as a resonator, or absorber, of incident acoustic energy seems to increase with depth. The condition that the swimbladder maintain a constant volume ensures that the mass of enclosed gas increases with ambient pressure, hence depth.

The results so far described have been for calculations of TS at single frequencies. However, as mentioned in Sec. V, sonar measurements use signals of finite duration and therefore possess bandwidth. In order to reproduce this characteristic more faithfully in the BEM predictions, further computations were undertaken in a frequency band around 38.1 $\mathrm{kHz}$ at the highest pressure of $51 \mathrm{~atm}$, where the effect of resonance is likely to be most significant. Equation (17) provides a basis for then determining a frequency-averaged TS. A spectral band of $\pm 1 \mathrm{kHz}$ about the center frequency was found to be sufficient to account for $95 \%$ of the scattered energy. Within this band, BEM predictions were made at intervals of $50 \mathrm{~Hz}$. The integral in the numerator of Eq. (17) was evaluated numerically, first as a Riemann summation, and second by fitting a cubic spline to the TS response and integrating using Simpson's rule with an interval of $10 \mathrm{~Hz}$. The results of the latter method were virtually identical to those of the former, the difference in the eventual value of $b$ in Eq. (18), for example, being generally less than $0.02 \mathrm{~dB}$.

The frequency-averaged results are included in Tables II and III, as the final line in each set of results for a given tilt distribution. Scatter diagrams and regression lines are plotted 
TABLE III. As Table II, but in ventral aspect.

\begin{tabular}{|c|c|c|c|c|c|c|}
\hline \multirow{2}{*}{$\begin{array}{c}\text { Frequency } \\
\text { kHz }\end{array}$} & \multicolumn{2}{|c|}{ Tilt angle (degrees) } & \multirow{2}{*}{$\begin{array}{c}\text { Pressure } \\
\text { (atm) }\end{array}$} & \multicolumn{3}{|c|}{ BEM computed TS functions } \\
\hline & Mean & s.d. & & $\rho$ & $b$ & SE \\
\hline 38.1 & 0.0 & 0.0 & 0 & 0.427 & -64.84 & 1.85 \\
\hline 38.1 & 0.0 & 0.0 & 1 & 0.425 & -64.84 & 1.84 \\
\hline 38.1 & 0.0 & 0.0 & 11 & 0.451 & -64.91 & 1.87 \\
\hline 38.1 & 0.0 & 0.0 & 51 & 0.494 & -64.88 & 1.89 \\
\hline $37.1-39.1$ & 0.0 & 0.0 & 51 & 0.453 & -64.79 & 1.75 \\
\hline 38.1 & 0.0 & 5.0 & 0 & 0.684 & -65.03 & 1.15 \\
\hline 38.1 & 0.0 & 5.0 & 1 & 0.682 & -65.03 & 1.14 \\
\hline 38.1 & 0.0 & 5.0 & 11 & 0.703 & -65.09 & 1.17 \\
\hline 38.1 & 0.0 & 5.0 & 51 & 0.653 & -65.12 & 1.43 \\
\hline $37.1-39.1$ & 0.0 & 5.0 & 51 & 0.736 & -64.95 & 1.02 \\
\hline 38.1 & 0.0 & 10.0 & 0 & 0.875 & -65.79 & 0.66 \\
\hline 38.1 & 0.0 & 10.0 & 1 & 0.874 & -65.79 & 0.66 \\
\hline 38.1 & 0.0 & 10.0 & 11 & 0.878 & -65.84 & 0.70 \\
\hline 38.1 & 0.0 & 10.0 & 51 & 0.823 & -65.83 & 0.93 \\
\hline $37.1-39.1$ & 0.0 & 10.0 & 51 & 0.907 & -65.69 & 0.57 \\
\hline 38.1 & -4.4 & 16.0 & 0 & 0.950 & -66.74 & 0.41 \\
\hline 38.1 & -4.4 & 16.0 & 1 & 0.948 & -66.74 & 0.41 \\
\hline 38.1 & -4.4 & 16.0 & 11 & 0.941 & -66.79 & 0.47 \\
\hline 38.1 & -4.4 & 16.0 & 51 & 0.875 & -66.74 & 0.74 \\
\hline $37.1-39.1$ & -4.4 & 16.0 & 51 & 0.952 & -66.62 & 0.39 \\
\hline
\end{tabular}

in Fig. 8 for the tilt distribution $\mathrm{N}(-4.4,16)^{\circ}$, allowing a direct comparison with Fig. 7.

In the dorsal aspect, the effects of averaging over the bandwidth of the signal are to slightly decrease the TS level as given by the value of $b$ in Eq. (18), increase the correlation, and decrease the variability when compared with the corresponding single-frequency results. In the ventral aspect, the TS level is slightly increased. The correlation is increased
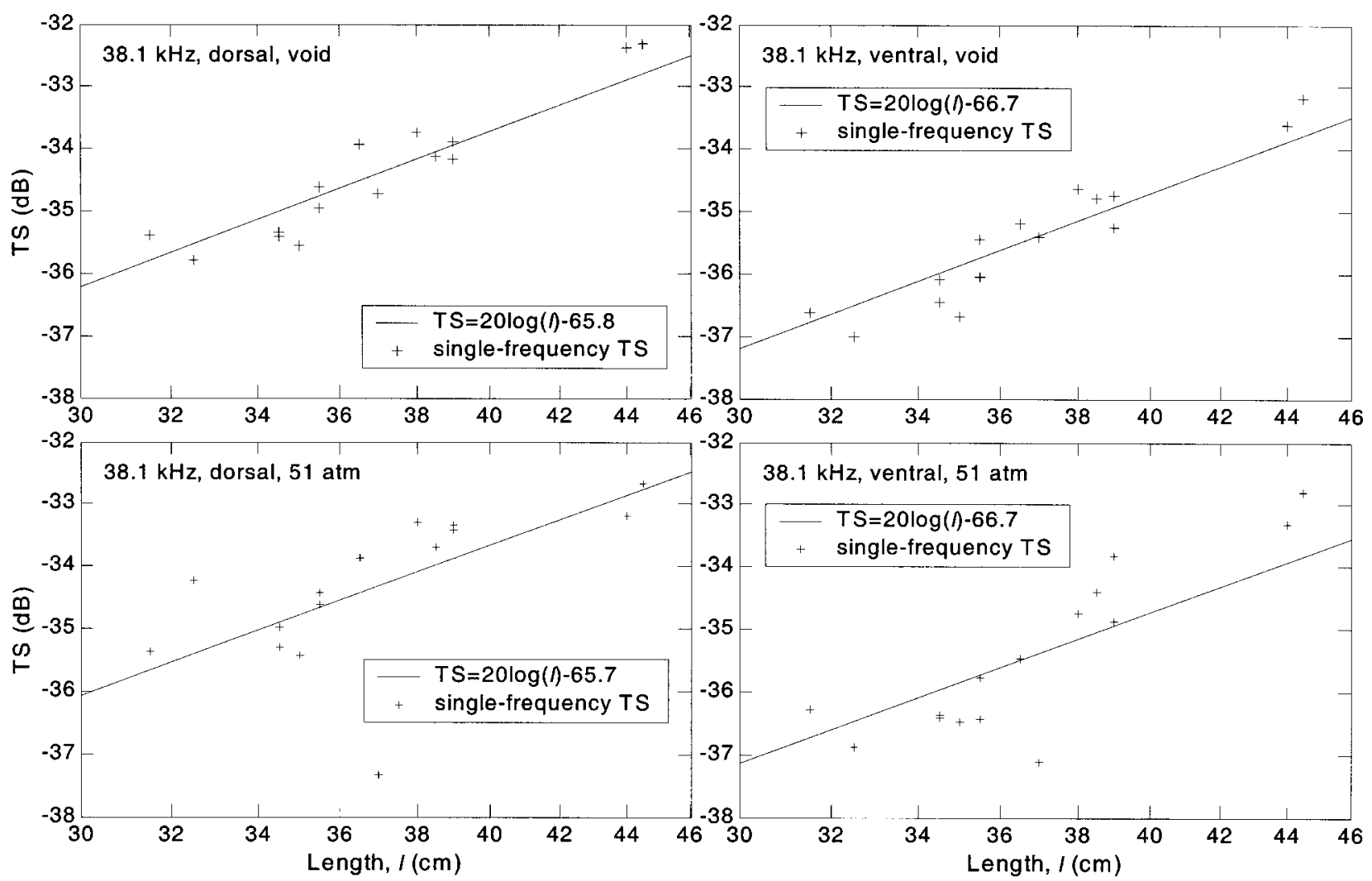

FIG. 7. Scatter diagram of target strength versus length $l$, expressed on a logarithmic scale, at the single frequency of $38.1 \mathrm{kHz}$ for the tilt distribution $(-4.4,16)$, for a void, and at a pressure of $51 \mathrm{~atm}$, for dorsal and ventral aspects. The regression equation TS $=20 \log l+b$ is shown by the continuous line in each case. 


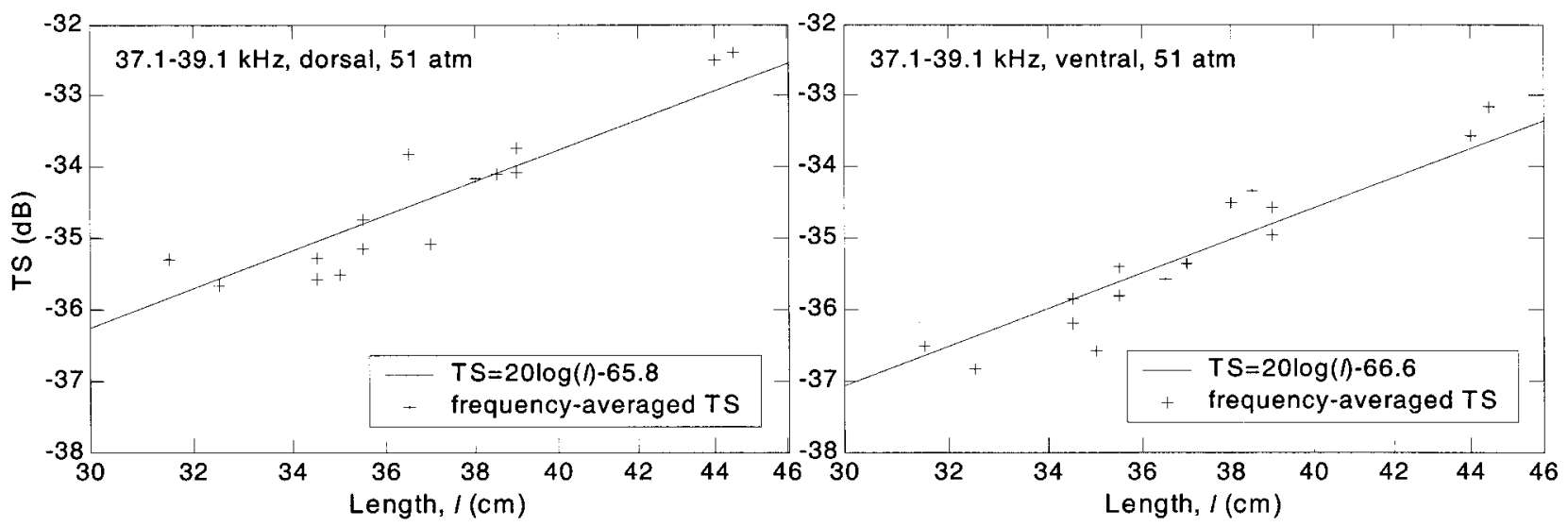

FIG. 8. As Fig. 7 at $51 \mathrm{~atm}$, but for target strength averaged over the frequency band $37.1-39.1 \mathrm{kHz}$, using Eq. (17).

in all cases but one, and the variability is decreased. These weak trends probably reflect the particular locations and magnitudes of resonance frequencies in the $2-\mathrm{kHz}$ band of averaging. The sample size of 15 specimens is simply too small, and the four orientation distributions are too dependent, to permit drawing stronger conclusions.

Since the depth dependence of target strength is weak, both the echo integration and echo counting methods can be applied as they are at present with at most minor corrections. More importantly, possible observed differences in in-situ target strength of depth-adapted gadoids may be attributed to behavior, as manifested through the orientation distribution, rather than to the simple effect of depth acting on the swimbladder volume.

\section{Future work}

Exercise of the BEM for the test case of a spherical gas-filled bubble revealed low- and high-frequency resonances, if without inclusion of internal damping. Only the lowest-frequency resonance seems to have been studied, suggesting future areas of experimental and theoretical investigation.

The size and shape of each swimbladder have been assumed to be constant, independent of depth. Insofar as the computations are meant to explore the depth dependence of target strength, these would describe situations in which the fish are fully depth-adapted, with inflated swimbladders. Under some circumstances of vertical migration, the rate of change in depth is so rapid that compensation by the rete mirabile lags behind, leading to situations of negative or positive buoyancy, as the fish is migrating respectively downwards or upwards. Examination of the change in target strength under uncompensated or partially compensated depth changes would be valuable, but requires a separate investigation. A prerequisite is knowledge of the manner of swimbladder form change with pressure change, which presently is mostly speculative.

Other swimbladder shapes and types should be amenable to computation with the BEM. A study of the Atlantic herring swimbladder, lacking rete mirabile, would be of particular value given that the fish is acoustically surveyed for purposes of estimating stock abundance.
Another problem waiting to be addressed is that of the induced surface velocity field on the swimbladder wall and its possible relationship to auditory function. The BEM has already been used in a preliminary study to generate the surface-velocity field ${ }^{41}$ and should shed light on the relationship of external acoustic stimuli and their transmission to the presumed organ of hearing.

\section{CONCLUSIONS}

The effect of depth on the target strength of depthadapted gadoids has been modeled by the boundary-element method. The mean target strength, based on the averaged backscattering cross section, has been found to change insignificantly with depth. There is, however, increased variability in the orientation dependence of target strength. In addition, it is evidently easier to excite high-frequency resonances with increasing depth.

Thus, for applications of the echo counting and integration methods ${ }^{6}$ in acoustic surveys of gadoid abundance, there need be no change either in execution of the surveys or in interpretation of forthcoming echo data. This assumes that the gadoid swimbladder remains fully inflated at all depths of measurement. In the event that depth excursions occur or are undertaken without concurrent compensation to maintain a constant state of inflation, the target strength may be expected to change, very possibly to a significant degree depending on the extent and rapidity of the depth excursion.

\section{ACKNOWLEDGMENTS}

This work began with sponsorship by the European Commission through its RTD-program, Contract No. MAS3CT95-0031 (BASS), and was completed with support by the Office of Naval Research, Contract No. N000140310368. Dr. P. J. Harris, University of Brighton, is thanked for discussions on the Burton-Miller formulation. Professor A. D. Pierce is thanked for discussions on the question of uniqueness. Dr. V. R. Starczak is thanked for assistance in executing preliminary BEM computations. A. Norton is thanked for much valued assistance in the technical editing. This is Woods Hole Oceanographic Institution Contribution No. 10839. 
${ }^{1}$ R. H. Love, "Measurements of fish target strength: a review," Fish. Bull. U.S. 69, 703-715 (1971).

${ }^{2}$ L. Midttun, "Fish and other organisms as acoustic targets," Rapp. P.-V. Reun.-Cons. Int. Explor. Mer 184, 25-33 (1984).

${ }^{3}$ K. G. Foote, "Summary of methods for determining fish target strength at ultrasonic frequencies," ICES J. Mar. Sci. 48, 211-217 (1991).

${ }^{4}$ S. McClatchie, G. Macaulay, R. F. Coombs, P. Grimes, and A. Hart, "Target strength of an oily deep-water fish, orange roughy (Hoplostethus atlanticus) I. Experiments," J. Acoust. Soc. Am. 106, 131-142 (1999).

${ }^{5}$ K. G. Foote, "Target strength of fish," in Encyclopedia of Acoustics, edited by M. J. Crocker (Wiley, New York, 1997), pp. 493-500.

${ }^{6}$ D. N. MacLennan, "Acoustical measurement of fish abundance," J. Acoust. Soc. Am. 87, 1-15 (1990).

${ }^{7}$ K. G. Foote, “Acoustic sampling volume," J. Acoust. Soc. Am. 90, 959964 (1991).

${ }^{8}$ O. Nakken and K. Olsen, "Target strength measurements of fish," Rapp. P.-V. Reun.-Cons. Int. Explor. Mer 170, 52-69 (1977).

${ }^{9}$ Y. Miyanohana, K. Ishii, and M. Furusawa, "Measurements and analyses of dorsal-aspect target strength of six species of fish at four frequencies," Rapp. P.-V. Reun.-Cons. Int. Explor. Mer 189, 317-324 (1990).

${ }^{10} \mathrm{~S}$. McClatchie, J. Alsop, and R. F. Coombs, "A re-evaluation of relationships between fish size, acoustic frequency, and target strength," ICES J. Mar. Sci. 53, 780-791 (1996).

${ }^{11}$ T. Mukai and K. Iida, "Depth dependences of target strength of live kokanee salmon in accordance with Boyle's Law," ICES J. Mar. Sci. 53, 245-248 (1996).

${ }^{12}$ K. Sawada, Z. Ye, R. Kieser, G. A. McFarlane, Y. Miyanohana, and M. Furusawa, "Target strength measurements and modeling of walleye pollock and Pacific hake," Fish. Sci. 65, 193-205 (1999).

${ }^{13}$ R. H. Love, "Resonant acoustic scattering by swimbladder-bearing fish," J. Acoust. Soc. Am. 64, 571-580 (1978).

${ }^{14}$ C. Feuillade and R. W. Nero, "A viscous-elastic swimbladder model for describing enhanced-frequency resonance scattering from fish," J. Acoust. Soc. Am. 103, 3245-3255 (1998).

${ }^{15}$ K. G. Foote, "Linearity of fisheries acoustics, with addition theorems," J. Acoust. Soc. Am. 73, 1932-1940 (1983).

${ }^{16} \mathrm{E}$. Ona, "Physiological factors causing natural variations in acoustic target strength of fish," J. Mar. Biol. Assoc. U.K. 70, 107-127 (1990).

${ }^{17}$ K. G. Foote and D. T. I. Francis, "Comparing Kirchhoff-approximation and boundary-element models for computing gadoid target strengths," J. Acoust. Soc. Am. 111, 1644-1654 (2002).

${ }^{18}$ L. H. Chen and D. G. Scheikert, "Sound radiation from an arbitrary body," J. Acoust. Soc. Am. 35, 1626-1632 (1963).

${ }^{19}$ G. Chertock, "Sound radiation from vibrating surfaces," J. Acoust. Soc. Am. 36, 1305-1313 (1964).

${ }^{20}$ L. G. Copley, "Integral equation method for radiation from vibrating bodies," J. Acoust. Soc. Am. 41, 807-816 (1967).

${ }^{21}$ L. G. Copley, "Fundamental results concerning integral representations in acoustic radiation," J. Acoust. Soc. Am. 44, 28-32 (1968).

${ }^{22}$ A. D. Pierce, "Variational formulations in acoustic radiation and scatter- ing," in Underwater Scattering and Radiation, edited by A. D. Pierce and R. N. Thurston (Academic, San Diego, 1992), pp. 195-371.

${ }^{23} \mathrm{H}$. A. Schenck, "Improved integral formulation for acoustic radiation problems," J. Acoust. Soc. Am. 44, 41-58 (1968).

${ }^{24}$ A. J. Burton and G. F. Miller, "The application of integral equation methods to the numerical solutions of some exterior boundary problems," Proc. R. Soc. London, Ser. A 323, 201-210 (1971).

${ }^{25}$ A. F. Seybert, B. Soenarko, F. J. Rizzo, and D. J. Shippy, "An advanced computational method for radiation and scattering of acoustic waves in three dimensions," J. Acoust. Soc. Am. 77, 362-368 (1985).

${ }^{26}$ T. W. Wu, A. F. Seybert, and G. C. Wan, "On the numerical implementation of a Cauchy principal value integral to insure a unique solution for acoustic radiation and scattering," J. Acoust. Soc. Am. 90, 554-560 (1991).

${ }^{27}$ T. W. Wu and A. F. Seybert, "A weighted residual formulation for the CHIEF method in acoustics," J. Acoust. Soc. Am. 90, 1608-1614 (1991).

${ }^{28}$ D. J. Segalman and D. W. Lobitz, "A method to overcome computational difficulties in the exterior acoustics problem," J. Acoust. Soc. Am. 91, 1855-1861 (1992).

${ }^{29}$ D. T. I. Francis, "A gradient formulation of the Helmholtz integral equation for acoustic radiation and scattering," J. Acoust. Soc. Am. 93, 17001709 (1993).

${ }^{30}$ W. L. Meyer, W. A. Bell, M. P. Stallybrass, and B. T. Zinn, "Prediction of the sound field radiated from axisymmetric surfaces," J. Acoust. Soc. Am. 65, 631-638 (1979).

${ }^{31} \mathrm{~T}$. Terai, "On calculation of sound fields around three dimensional objects by integral equation methods," J. Sound Vib. 69, 71-100 (1980).

${ }^{32}$ K. A. Cunefare and G. Koopman, "A boundary element method for acoustic radiation valid for all wavenumbers," J. Acoust. Soc. Am. 85, 39-48 (1989).

${ }^{33}$ O. C. Zienkiewicz and R. L. Taylor, The Finite Element Method, 4th ed. (McGraw-Hill, London, 1989), Vol. 1.

${ }^{34}$ V. C. Anderson, "Sound scattering from a fluid sphere," J. Acoust. Soc. Am. 22, 426-431 (1950).

${ }^{35}$ R. R. Goodman and R. Stern, "Reflection and transmission of sound by elastic spherical shells," J. Acoust. Soc. Am. 34, 338-344 (1962).

${ }^{36}$ K. G. Foote, "Rather-high-frequency sound scattering by swimbladdered fish,” J. Acoust. Soc. Am. 78, 688-700 (1985).

${ }^{37} \mathrm{~K}$. Olsen, "Orientation measurements of cod in Lofoten obtained from underwater photography, and their relation to target strength," Counc. Meet. Int. Counc. Explor. Sea 1971/B:17.

${ }^{38}$ K. G. Foote, "Averaging of fish target strength functions," J. Acoust. Soc. Am. 67, 504-515 (1980).

${ }^{39}$ National Defense Research Committee, "Physics of sound in the sea" (Department of the Navy, Washington, D.C., 1969), Chap. 28.

${ }^{40} \mathrm{~K}$. G. Foote, "Optimizing copper spheres for precision calibration of hydroacoustic equipment," J. Acoust. Soc. Am. 71, 742-747 (1982).

${ }^{41}$ D. T. I. Francis and K. G. Foote, "Acoustic excitation of the fish swimbladder," Bioacoustics 12, 265-267 (2002). 